\title{
Development of a Dynamic Multi-Protein Signature of Postoperative Delirium
}

\author{
Sarinnapha M. Vasunilashorn, PhD, ${ }^{1,2,3}$ Long H. Ngo, PhD, ${ }^{1,2}$ Noel Y. Chan, PhD, ${ }^{2,4,5}$ \\ Wenxiao Zhou, MSc, ${ }^{1}$ Simon T. Dillon, PhD, ${ }^{2,4,5}$ Hasan H. Otu, PhD, ${ }^{6}$ \\ Sharon K. Inouye, MD, MPH, ${ }^{2,3,7}$ Iris Wyrobnik, BS, ${ }^{4,5}$ George A. Kuchel, MD, ${ }^{8}$ \\ Janet E. McElhaney, MD, ${ }^{9}$ Zhongcong Xie, MD, PhD, ${ }^{2,10}$ David C. Alsop, PhD, ${ }^{2,11}$ \\ Richard N. Jones, ScD, ${ }^{3,12}$ Towia A. Libermann, PhD, ${ }^{2,4,5, *}$ and \\ Edward R. Marcantonio, MD, SM12,2,3,7
}

'Division of General Medicine and Primary Care, Department of Medicine, Beth Israel Deaconess Medical Center (BIDMC), Boston, Massachusetts. ${ }^{2}$ Harvard Medical School, Boston, Massachusetts. ${ }^{3}$ Aging Brain Center, Institute for Aging Research, Hebrew SeniorLife, Boston, Massachusetts. ${ }^{4}$ Division of Interdisciplinary Medicine and Biotechnology, BIDMC, Boston, Massachusetts. ${ }^{5}$ BIDMC Genomics, Proteomics, Bioinformatics and Systems Biology Center, Boston, Massachusetts. ${ }^{6}$ Department of Electrical and Computer Engineering, University of Nebraska-Lincoln. 'Division of Gerontology, BIDMC, Boston, Massachusetts. ${ }^{8}$ University of Connecticut Center on Aging, University of Connecticut Health Center, Farmington. ${ }^{9} \mathrm{Health}$ Sciences North Research Institute, Sudbury, Ontario, Canada. ${ }^{10} \mathrm{Department}$ of Anesthesia, Critical Care and Pain Medicine, Massachusetts General Hospital, Boston. "1Department of Radiology, BIDMC, Boston, Massachusetts. ${ }^{12}$ Department of Psychiatry and Human Behavior, Warren Alpert Medical School, Brown University, Providence, Rhode Island.

${ }^{*}$ Co-senior authors.

Address correspondence to: Sarinnapha M. Vasunilashorn, PhD, Division of General Medicine and Primary Care, Beth Israel Deaconess Medical Center, 1309 Beacon Street, Brookline, MA 02446. E-mail: svasunil@bidmc.harvard.edu

Received: September 14, 2017; Editorial Decision Date: February 5, 2018

Decision Editor: Anne Newman, MD, MPH

\begin{abstract}
Background: Delirium is common, morbid, and costly, yet its biology is poorly understood. We aimed to develop a multi-protein signature of delirium by identifying proteins associated with delirium from unbiased proteomics and combining them with delirium biomarkers identified in our prior work (interleukin [IL]-6 and IL-2).

Methods: We used the Successful Aging after Elective Surgery (SAGES) Study of adults age $\geq 70$ undergoing major noncardiac surgery $(N=560 ; 24 \%$ delirium). Plasma was collected preoperatively (PREOP) and on postoperative day 2 (POD2). In a nested matched case-control study involving 12 pairs of delirium cases and no-delirium controls, isobaric tags for relative and absolute quantitation-based (iTRAQ) mass spectrometry proteomics was applied to identify the top set of delirium-related proteins. With these proteins, we then conducted enzymelinked immunosorbent assay (ELISA) confirmation, and if confirmed, ELISA validation in 75 matched pairs. Multi-marker conditional logistic regression was used to select the "best" PREOP and POD2 models for delirium.

Results: We identified three proteins from iTRAQ: C-reactive protein (CRP), zinc alpha-2 glycoprotein (AZGP1), and alpha-1 antichymotrypsin (SERPINA3). The "best" multi-protein models of delirium included: PREOP: CRP and AZGP1 (Bayesian information criteria [BIC]: 93.82, c-statistic: 0.77); and POD2: IL-6, IL-2, and CRP (BIC: 87.11, c-statistic: 0.84).

Conclusion: The signature of postoperative delirium is dynamic, with some proteins important before surgery (risk markers) and others at the time of delirium (disease markers). Our dynamic, multi-protein signature for delirium improves our understanding of delirium pathophysiology and may identify patients at-risk of this devastating disorder that threatens independence of older adults.
\end{abstract}

Keywords: Delirium, Proteomics, Protein signature 
Delirium, an acute confusional state, is characterized by fluctuations in attention, thinking, and consciousness. It affects $15 \%-53 \%$ of older surgical patients $(1,2)$, and is associated with several adverse outcomes, including greater postoperative complications, longer hospital stay, higher discharge to a nursing home, higher in-hospital mortality, and in the long term, higher risk of incident Alzheimer's disease $(\mathrm{AD})(3,4)$. Although knowledge of delirium epidemiology continues to expand, its pathophysiology is less understood.

Several biological models of delirium have been hypothesized (5), one of which is the neuroinflammatory model (2). Our recent work has supported a related inflammatory model of postoperative delirium. Using a multiplex Luminex panel, we found interleukin (IL)-6 levels measured on postoperative day 2 (POD2) and IL-2 levels measured preoperatively (PREOP) and on POD2 were elevated in delirium cases relative to their matched no-delirium controls (6). Additionally, using an unbiased proteomics approach followed by enzyme-linked immunosorbent assay (ELISA) validation, we identified inflammatory marker C-reactive protein (CRP) as the top protein associated with postoperative delirium at PREOP and POD2 (7). Despite the growing evidence in support of an inflammatory model of delirium $(2,5,8,9)$, we acknowledge that other pathophysiological pathways may be involved.

Since completing the above work, we have conducted additional proteomics experiments and validation analyses, and several additional proteins besides CRP have emerged, suggesting that other potential biological pathways aside from the neuroinflammatory pathway may be important in delirium. The current study significantly extends our previous work by: (i) identification and reporting of novel proteins associated with postoperative delirium using an unbiased proteomics methodology followed by ELISA confirmation and validation, and (ii) development of new and superior multi-protein predictive models for postoperative delirium combining these newly discovered proteins with those discovered in our previous work.

\section{Methods}

\section{Study Population}

The Successful Aging after Elective Surgery (SAGES) study is an ongoing prospective cohort study aimed at understanding risk factors and long-term outcomes of delirium. The study design and methods have been previously described $(10,11)$. Briefly, the SAGES study enrolled patients without preoperative delirium age $\geq 70$ who were scheduled for major noncardiac surgery $(N=560)$, including orthopedic, vascular, or colectomy-under general or spinal anesthesia. Patients with dementia were excluded based on a detailed screening process (detailed in $(10,11)$ ), which culminated in a neurocognitive battery at baseline, which was used to compute the general cognitive performance (GCP) summary measure (12). Comorbidities were identified from medical record review. Informed consent for study participation was obtained from all subjects according to procedures approved by the institutional review boards of Beth Israel Deaconess Medical Center and Brigham and Woman's Hospital, the two surgical sites, and Hebrew SeniorLife, the study coordinating center, all located in Boston, Massachusetts.

\section{Specimen Collection}

All patients underwent phlebotomy at four time points: PREOP, postanesthesia care unit (PACU), POD2, and 1 month postop (PO1MO). Based on previous findings, the current study focuses on the PREOP and POD2 time points due to their paramount importance for identifying risk markers and disease markers of delirium, respectively
(13). Blood collection was incorporated into clinical blood draws taken in the pre-admitting testing center (PREOP) and on the surgical wards (POD2). Mechanical disruption during phlebotomy was minimized to prevent hemolysis, and blood was stored on ice in heparinized tubes until processing. We used low-speed centrifugation $\left(1500 \mathrm{~g}\right.$ for 15 minutes at $\left.4^{\circ} \mathrm{C}\right)$ to separate plasma and cellular material, and plasma was stored at $-80^{\circ} \mathrm{C}$ until analysis.

\section{Delirium and Subsyndromal Delirium}

Postoperative delirium was determined from daily interviews during hospitalization, supplemented with a validated chart review method, used to maximize sensitivity (14). All interviewers underwent training to conduct structured mental status examinations that tested attention, orientation, and memory. Delirium was assessed using the Confusion Assessment Method (CAM) diagnostic algorithm, which required the patient to have an acute onset of change or fluctuating mental status, inattention, and either disorganized thinking or altered level of consciousness (15). The presence of delirium by chart review was adjudicated by at least two delirium experts, and discordance was resolved through consensus. Patients were considered delirious if delirium was present on either the CAM or the chart review method on any postoperative day; otherwise, patients were considered nondelirious (15). If delirium was absent during the entire hospitalization, subsyndromal delirium was defined as (i) an acute change in mental status or fluctuation, (ii) at least one CAM core feature (inattention, disorganized thinking, altered level of consciousness), and (iii) at least one other CAM supporting feature (disorientation, perceptual disturbance, delusion, psychomotor agitation, psychomotor retardation, or inappropriate behavior).

\section{Samples for ITRAQ Discovery and ELISA Confirmation and Validation}

Two subgroups from the 560 SAGES participants were used to examine the association between proteins and delirium: (i) the full matched pair sample of 75 matched delirium-cases/no-delirium control pairs [previously described $(6,7,16)]$, and (ii) the iTRAQ matched pair sample of 12 matched pairs randomly selected from the overall full matched pair sample.

Cases and controls were matched on six variables thought to be important in postoperative delirium [previously described (6)]: age within 5 years; baseline GCP within 5 points; and an exact match for sex, surgery type, presence of vascular comorbidity, and Apolipoprotein E $\varepsilon 4$ carrier status.

Delirium cases were defined as participants with either: (i) delirium on POD2; or (ii) delirium on POD1 and subsyndromal delirium on POD2 or POD3. No-delirium controls were defined as patients without delirium or without subsyndromal delirium on any postoperative day, ensuring that controls did not include patients with any symptoms of delirium. Detailed information on the inclusion/exclusion criteria and matching methods have been described previously $(6,7,14)$.

\section{Proteomics Analysis}

Our protein biomarker discovery and validation methods were conducted in four stages (Figure 1). In Stage 1, we performed unbiased proteomics on abundant protein depleted plasma using 8-plex isobaric tags for relative and absolute quantitation mass spectrometry (iTRAQ; 17) to examine 12 sets of matched case-control samples, as previously described (7). Each experiment involved our 8-plex iTRAQ labeling of one case-control pair at four time points (ie, we conducted 12 experiments). 


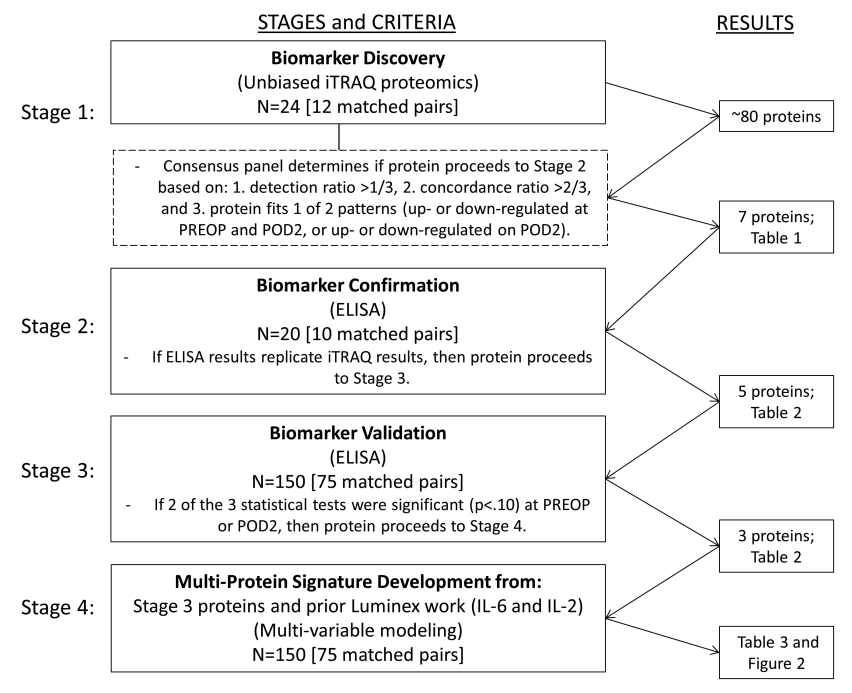

Figure 1. Methodologic steps for protein identification and ELISA confirmation and validation of proteins for determination of final multiprotein signature of postoperative delirium. ELISA = enzyme-linked immunosorbent assay; IL = interleukin; ITRAQ = isobaric tags for relative and absolute quantitation-based relative quantitation; POD2 = postoperative day 2; PREOP = preoperative.

To assay lower abundance proteins, the most dominant proteins were depleted using an antibody-based depletion column (MARS-14 column [Agilent, Santa Clara, CA] or Genway top-14 column coupled with the Supermix column [Sigma]) following each manufacturer's recommended protocol. Depletion columns were connected to an Agilent 1200 high-performance liquid chromatography (HPLC) and unbound protein flow-through was collected and retained for further analysis. Ice-cold acetone precipitation was used to remove extraneous nonprotein primary amine groups. After resolubilization in an iTRAQ compatible buffer, $100 \mu \mathrm{g}$ of protein/sample was reduced, alkylated and then digested overnight with trypsin. The resultant peptides were labeled with iTRAQ tags. All eight iTRAQ-labeled samples per run were pooled, fractionated by two-dimensional HPLC (LC/LC) and peptides identified by tandem mass spectrometry (MS/MS) on a 4800 MALDI-TOF/TOF mass spectrometer (ABSciex, Framingham, MA). Peptide and protein detection and differential expression analysis were performed using the ProteinPilot v4.5b software (ABSciex).

Across all 12 iTRAQ experiments an average of 83 proteins were detected per experiment at an Unused ProtScore of 1.3 or larger $(95 \%$ confidence in detection $[p<.05])$. We identified dysregulated proteins between cases and controls based on the ratio of the protein expression levels in cases and controls $(p<.05)$.

In assessing the data from the 12 matched pair iTRAQ proteomics runs, a consensus panel (author initials: SMV, LHN, WZ, STD, HHO, TAL, ERM) adopted the following three criteria to rank and select proteins for ELISA confirmation (Stage 2): (i) detection rate $>1 / 3$, defined as the fraction of the samples (ie, $>4 / 12$ matched pairs) in which the protein was detected with a detection $p$-value $<$ .05 ; (ii) concordance rate $>2 / 3$, defined as the fraction of the samples in which the protein was detected with $95 \%$ confidence and with concordant expression (upregulated or downregulated) in cases versus controls; and (iii) protein must fit one of two patterns: (i) upregulated [or downregulated] at PREOP and POD2, characteristic of a risk marker; or (ii) upregulated [or downregulated] on POD2, characteristic of a disease marker (13). Proteins at PREOP and proteins at POD2 were independently selected for ELISA confirmation
(Stage 2) if they met our selection criteria and a high-quality ELISA kit was commercially available.

\section{ELISA Confirmation and Validation}

During Stage 2, ELISA confirmation of the top proteins was conducted using 10 randomly selected matched pairs from the iTRAQ Matched Pair Sample (Stage 1). Ten out of 12 pairs were used because only 40 samples from 10 pairs at 2 time points (case/control at PREOP and POD2) can be run in duplicate on a single 96-well ELISA plate, allowing the remaining 16 wells for the standard curve. If results from ELISA confirmation replicated the iTRAQ findings, then the protein proceeded to ELISA validation (Stage 3). In the case of equivocal findings after 10 matched pairs, confirmation on an additional 10 matched pairs was performed, and re-evaluated for confirmation of iTRAQ findings.

In Stage 3, three tests of significance were adopted to evaluate ELISA validation in the full matched pair sample of 75 matched casecontrol pairs: (i) binomial tests: evaluates consistency of directionality (upregulated vs downregulated); (ii) paired $t$-tests: compares mean protein level differences in cases vs controls (standardized to mean $=0$, standard deviation $=1$ ); and (iii) signed rank tests: compares rank of protein level differences to consider non-normal distributions of the paired differences $(7,8)$. To be conservative, proteins with $p$-values $<.10$ for at least two of the three tests at either the PREOP or POD2 time point were considered validated by ELISA. ELISAs from R\&D Systems (Minneapolis, $\mathrm{MN}$ ) were used when available; otherwise, an alternate US or European-based manufacturer was selected (Genway Biotech [San Diego, CA], AdipoGen Life Sciences [Liestal, Switzerland]).

Stage 4 identified the final proteins for incorporation in the multi-protein signature for postoperative delirium. We incorporated ELISA-validated proteins from Stage 3 with biomarkers associated with postoperative delirium from our previous work $(6,7)$, and used multivariable modeling to select the "best" PREOP and "best" POD2 analytic models. Specifically, we used conditional logistic regression and evaluated: (i) model fit and parsimony using Bayesian information criteria (BIC) (18), and (ii) the c-statistic to measure ability to distinguish cases from controls (area under the receiver operating characteristic [ROC] curve). The "best" analytic model for postoperative delirium at each time point (PREOP and POD2) was selected based on the lowest $\mathrm{BIC}$ and highest c-statistic, with priority given to the lowest BIC. We computed odds ratios (ORs) and 95\% confidence intervals for each protein marker within the "best" analytic model for PREOP and POD2. To explore possible collinearity among all proteins considered in the analytic models, we examined their resulting Spearman correlation coefficients $(r)$. All analyses were conducted using SAS software version 9.3 (SAS Institute, Cary, NC).

\section{Results}

The sample characteristics of the match variables for the cases and controls in the full 75 matched pair sample were generally similar, and have been previously reported (6,7). Briefly, cases and controls were on average 77 years old, $56 \%$ female, and the prevalence of orthopedic, vascular, and gastrointestinal surgeries was $88 \%, 5 \%$, and $7 \%$ (respectively).

\section{Stage 1: Biomarker Discovery}

Table 1 lists the 19 PREOP and 26 POD2 proteins from iTRAQ that met our criteria of $>1 / 3$ detection rate, and $>2 / 3$ concordance rate in distinguishing cases from controls. From this list, the seven proteins selected from our consensus voting process for ELISA confirmation 
Table 1. Identification of Top* PREOP and POD2 Proteins from iTRAQ

\begin{tabular}{|c|c|c|c|c|}
\hline & Concordance Rate (\%) & Detection Rate (\%) & No. of Up-regulated & $\begin{array}{l}\text { No. of Down- } \\
\text { regulated }\end{array}$ \\
\hline \multicolumn{5}{|l|}{ PREOP } \\
\hline Histidine-rich glycoprotein (HRG) & 88 & 67 & 7 & 1 \\
\hline Alpha-1B-glycoprotein (A1BG) & 88 & 67 & 1 & 7 \\
\hline Fibrinogen alpha chain (FIBA) & 88 & 67 & 1 & 7 \\
\hline Zinc-alpha-2-glycoprotein (AZGP1) & 83 & 100 & 2 & 10 \\
\hline Complement $\mathrm{C} 2(\mathrm{CO} 2)$ & 80 & 42 & 1 & 4 \\
\hline Fibrinogen beta chain (FIBB) & 80 & 42 & 1 & 4 \\
\hline Hemopexin (HPX) & 78 & 75 & 2 & 7 \\
\hline Complement component C9 (CO9) & 75 & 67 & 6 & 2 \\
\hline ITIH2 & 75 & 67 & 6 & 2 \\
\hline Antithrombin-III (ANT3) & 75 & 67 & 2 & 6 \\
\hline Retinol-binding protein 4 (RET4) & 75 & 67 & 2 & 6 \\
\hline IGFALS & 75 & 67 & 2 & 6 \\
\hline Serotransferrin (TRFE) & 75 & 33 & 3 & 1 \\
\hline Complement C4-B (CO4B) & 75 & 33 & 3 & 1 \\
\hline Thyroxine-binding globulin (THBG) & 75 & 33 & 3 & 1 \\
\hline Extracellular matrix protein 1 (ECM1) & 75 & 33 & 1 & 3 \\
\hline Complement factor I (CFAI) & 67 & 75 & 3 & 6 \\
\hline C-reactive protein (CRP) & 67 & 50 & 4 & 2 \\
\hline CBPN & 67 & 50 & 2 & 4 \\
\hline \multicolumn{5}{|l|}{ POD2 } \\
\hline CRP & 100 & 50 & 6 & 0 \\
\hline Haptoglobin (HP) & 100 & 42 & 0 & 5 \\
\hline ECM1 & 100 & 33 & 0 & 4 \\
\hline Plasminogen (PLMN) & 88 & 67 & 7 & 1 \\
\hline Apolipoprotein A-IV (APOA4) & 80 & 83 & 2 & 8 \\
\hline Alpha-1-acid glycoprotein 1 (ORM1) & 80 & 42 & 4 & 1 \\
\hline HPX & 78 & 75 & 2 & 7 \\
\hline Clusterin (CLUS) & 75 & 100 & 9 & 3 \\
\hline AZGP1 & 75 & 100 & 3 & 9 \\
\hline HRG & 75 & 67 & 6 & 2 \\
\hline Fibrinogen alpha chain (FIBA) & 75 & 67 & 6 & 2 \\
\hline $\mathrm{CO} 4 \mathrm{~B}$ & 75 & 33 & 1 & 3 \\
\hline Complement factor D (CFAD) & 75 & 33 & 1 & 3 \\
\hline TRFE & 75 & 33 & 1 & 3 \\
\hline Complement C4-A (CO4A) & 75 & 33 & 1 & 3 \\
\hline Hyaluronan-binding protein 2 (НАBР2) & 75 & 33 & 3 & 1 \\
\hline Carboxypeptidase B2 (CBPB2) & 72 & 58 & 2 & 5 \\
\hline IBP3 & 70 & 83 & 3 & 7 \\
\hline Alpha-1-antichymotrypsin (SERPINA3) & 67 & 100 & 8 & 4 \\
\hline
\end{tabular}

Notes: CBPN = carboxypeptidase $\mathrm{N}$ catalytic chain; IBP3 = insulin-like growth factor-binding protein 3; IGFALS = insulin-like growth factor-binding protein complex acid labile subunit; ITIH2 = inter-alpha-trypsin inhibitor heavy chain H2; iTRAQ = isobaric tag for relative and absolute quantitation; POD2 = postoperative day 2; PREOP = preoperative. $N=24$ (12 matched pairs). No. of upregulated and downregulated are dependent on the detection rate. Proteins highlighted in the dark gray indicate the four selected proteins for ELISA confirmation that appear in both the PREOP and POD2 lists. Proteins highlighted in light gray indicate the three selected proteins for ELISA confirmation that appear only in the POD2 list.

"Top proteins were defined as proteins with (i) detection rate $>1 / 3(33.3 \%)$ and (ii) concordance rate $>2 / 3(66.7 \%)$.

included four proteins appearing in the lists for both PREOP and POD2, the risk marker pattern (CRP, hemopexin [HPX], zinc-alpha2-glycoprotein [AZGP1], and histidine-rich glycoprotein [HRG]); and three proteins appearing only on the POD2 list, the disease marker pattern (haptoglobin [HP], alpha-1-acid glycoprotein 1 [ORM1], and alpha-1-antichymotrypsin [SERPINA3]). HPX and AZGP1 were generally down-regulated in delirium; in contrast, CRP, HRG, HP, ORM1, and SERPINA3 were generally up-regulated in delirium.

\section{Stage 2: Biomarker Confirmation}

Among these seven proteins, ELISA analysis of the 10 matched pairs confirmed an association with delirium similar to the iTRAQ results for five proteins: CRP, SERPINA3, HPX, ORM1, and AZGP1. In these analyses, HRG and HP did not yield evidence of an association with delirium and were dropped from further consideration.

\section{Stage 3: Biomarker Validation}

Table 2 reports the ELISA findings for these five proteins in the full 75 matched pair sample. The three proteins that met our criteria for ELISA validation included: CRP (all three tests significant at PREOP and POD2), SERPINA3 (paired $t$-test and signed-rank test significant on POD2), and AZGP1 (all three tests significant at both time points). HPX and ORM1 did not meet criteria for validation and were dropped from further consideration. 
Table 2. Results From ELISA Validation (mg/L) of the Proteins From iTRAQThat Met ELISA Confirmation

\begin{tabular}{|c|c|c|c|c|c|c|c|c|c|}
\hline & Up-reg & Down-reg & $\begin{array}{l}p \text {-Value } \\
\text { Binomial }\end{array}$ & $\begin{array}{l}\text { Mean } \pm \text { SD for } \\
\text { Case }\end{array}$ & $\begin{array}{l}\text { Mean } \pm \text { SD for } \\
\text { Control }\end{array}$ & $\begin{array}{l}p \text {-Value } \\
\text { Paired } t \text {-Test }\end{array}$ & $\begin{array}{l}\text { Mean } \pm \text { SD of } \\
\text { Pair Differences }\end{array}$ & $\begin{array}{l}\text { Median (Range) of } \\
\text { Pair Differences }\end{array}$ & $\begin{array}{l}p \text {-Value } \\
\text { Signed Rank } \\
\text { Test }\end{array}$ \\
\hline \multicolumn{10}{|l|}{ PREOP } \\
\hline CRP & 48 & 27 & 0.01 & $7.2 \pm 8.4$ & $3.5 \pm 5.6$ & $<.01$ & $3.7 \pm 10.7$ & $1.6(-1.6,7.9)$ & $<.01$ \\
\hline HPX & 40 & 35 & 0.32 & $1210.2 \pm 285.3$ & $1299.4 \pm 524.3$ & 0.06 & $-89.2 \pm 412.2$ & $13.0(-185.8,113.0)$ & 0.46 \\
\hline ORM1 & 44 & 31 & 0.08 & $859.4 \pm 324.6$ & $784.3 \pm 299.1$ & 0.13 & $75.2 \pm 427.5$ & $40.0(-222.0,303.0)$ & 0.22 \\
\hline AZGP1 & 25 & 50 & 0.00 & $36.0 \pm 8.5$ & $40.3 \pm 10.0$ & 0.00 & $-4.2 \pm 12.6$ & $-2.8(-11.0,3.1)$ & 0.00 \\
\hline \multicolumn{10}{|l|}{ POD2 } \\
\hline CRP & 53 & 22 & $<0.01$ & $190.5 \pm 62.5$ & $140.8 \pm 64.8$ & $<0.01$ & $49.7 \pm 93.2$ & $63.8(-22.3,126.2)$ & $<.01$ \\
\hline SERPINA3 & 41 & 34 & 0.24 & $528.2 \pm 249.7$ & $480.5 \pm 198.5$ & 0.05 & $47.8 \pm 210.8$ & $16.7(-66.9,172.6)$ & 0.07 \\
\hline HPX & 41 & 34 & 0.24 & $1002.2 \pm 277.9$ & $1007.8 \pm 251.0$ & 0.82 & $-5.6 \pm 213.0$ & $17.0(-135.0,136.1)$ & 0.87 \\
\hline ORM1 & 37 & 38 & 0.59 & $1306.2 \pm 347.9$ & $1287.3 \pm 325.3$ & 0.69 & $18.9 \pm 409.8$ & $-9.0(-234.0,254.0)$ & 0.69 \\
\hline AZGP1 & 28 & 47 & 0.02 & $29.9 \pm 7.3$ & $32.7 \pm 7.7$ & 0.02 & $-2.77 \pm 9.9$ & $-3.6(-9.0,3.5)$ & 0.01 \\
\hline
\end{tabular}

Note: SERPINA3 = alpha-1 antichymotrypsin (Genway Biotech); ORM1 = alpha-1 glycoprotein (R\&D Systems); CRP = C-reactive protein (R\&D Systems); down-reg = downregulated; HPX = hemopexin (Genway Biotech); POD2 = postoperative day 2; PREOP = preoperative; up-reg = upregulated; AZGP1 = zincalpha2-glycoprotein (AdipoGen Life Sciences). $N=150$ (75 matched pairs). If two of the three statistical tests were significant ( $p<.10$, bolded) at PREOP or POD2, then protein was considered validated by ELISA. Our correlation analyses indicated that CRP was moderately correlated with: SERPINA3 at PREOP and POD2 $(r=0.41$ and 0.34 , respectively, $p<.0001$ for both $)$, and IL-6 $(r=0.46, p<.0001)$.

\section{Stage 4: Multi-Protein Signature Development}

To examine the individual and combined value of these three proteins (CRP, SERPINA3, and AZGP1) with markers associated with delirium in our previous work (IL-6 and IL-2), we examined all possible combinations of models among these five markers. This resulted in 31 total models consisting of: one marker (5 models), two markers (10 models), three markers (10 models), four markers ( 5 models), and five markers ( 1 model). Table 3 presents the model fit statistics for each model. Among the models with $>1$ marker, the model with CRP and AZGP1 was the "best combined model" for PREOP (BIC: 90.28, c-statistic: 0.77), and the model with IL-6, IL-2, and CRP was the "best combined model" for POD2 (BIC: 81.31, c-statistic: 0.83 ). Of note, the c-statistics for both of these multi-protein signatures are substantially higher than the best c-statistics for any single protein model. Additionally, the component proteins in the best signatures at PREOP and POD2 differ, suggesting that the protein signature for delirium is "dynamic" (changes over time).

Figure 2 illustrates the ORs and 95\% CIs for the models of each individual protein and the models for the "best combined model" for PREOP (Figure 2A) and POD2 (Figure 2B). In the individual and best combined PREOP models (Figure 2A), lower levels of AZGP1 and higher levels of CRP were associated with a higher risk of delirium. In the individual and best combined POD2 models, higher levels of IL-6, IL-2, and CRP were associated with a higher risk of delirium.

\section{Discussion}

This study used quantitative mass-spectrometry proteomics, followed by ELISA confirmation and validation to identify and validate proteins associated with delirium both preoperatively and on POD2. We found strong evidence for the involvement of CRP, AZGP1, and SERPINA3. Using these proteins along with biomarkers identified in our previous work [IL-6 and IL-2 (6)], we conducted multivariable modeling and identified a dynamic multi-protein signature of delirium, characterized by: (i) AZGP1 and CRP at PREOP, and (ii) IL-6, IL-2, and CRP at POD2. Based on model fit statistics, these PREOP and POD2 multi-protein signatures provide improved prediction of postoperative delirium compared to any single protein model of delirium.

Our findings confirm and substantially extend previous work examining the associations of inflammation and delirium. Higher postoperative levels of IL-6 at POD2 and IL-2 at PREOP and POD2 were previously associated with delirium in the same 75 matched pairs of the full matched pair sample used in the current study (6). Additionally, prior studies have underscored the role of CRP in delirium pathophysiology [eg, (19)], including findings from a subsample of 75 matched delirium cases and no-delirium controls (7). The latter project (7), which used a similar approach as the current study, aimed to discover the single top protein associated with delirium. The current study extends this work by identifying and validating additional proteins associated with delirium: AZGP1 and SERPINA3, and combining proteins into a multi-protein signature.

In contrast to the increased expression of IL-6, IL-2, CRP, and SERPINA3 in patients with delirium, AZGP1 is the first protein identified to be lower in plasma of patients who develop delirium. AZGP1, a single-chain polypeptide secreted by epithelial cells from the liver and other organs as well as adipocytes and found in various body fluids, participates in several important physiological functions, including stimulation of lipolysis (20). A proteomics approach of 53 PREOP cerebrospinal fluid (CSF) samples from older hip fracture patients identified increased expression of AZGP1 in patients with postoperative delirium relative to patients without delirium, the opposite of what we determined here in plasma; however, these associations in CSF were not replicated by ELISA validation (21). It may be that PREOP levels of plasma-based AZGP1 are involved in delirium pathophysiology, but that CSF levels of AZGP1 play a less prominent role.

Reduced expression of AZGP1 in plasma of patients at PREOP who develop delirium fits well with our model of a pre-inflammatory state in patients at-risk of developing delirium. There is strong evidence that macrophage-associated inflammation and the pro-inflammatory cytokine, TNF- $\alpha$, lead to a significant decrease in AZGP1 expression in fat and liver (22), and early sepsis is also linked to reduction in AZGP1 expression (23). Future studies are required to further probe the role of AZGP1 in delirium pathophysiology and as a therapeutic target. 
Table 3. Model Fit Statistics for All Possible Analytic Models That Consider the Individual and Combined Contributions of CRP, IL-6, IL-2, SERPINA3, and AZGP1

\begin{tabular}{|c|c|c|c|c|c|c|c|c|}
\hline & & & & & PREOP & & POD2 & \\
\hline Bioma & sinclu & $n$ the $m$ & & & $\mathrm{BIC}$ & C-Statistic $(95 \% \mathrm{CI})$ & $\mathrm{BIC}$ & C-Statistic $(95 \% \mathrm{CI})$ \\
\hline 1 Bion & & & & & & & & \\
\hline & & & & AZGP1 & 99.71 & $0.70(0.58-0.82)$ & 102.17 & $0.67(0.54-0.79)$ \\
\hline & & & $\mathrm{A} 1 \mathrm{AC}$ & & 106.62 & $0.58(0.47-0.69)$ & 104.04 & $0.62(0.50-0.75)$ \\
\hline & & CRP & & & 98.45 & $0.69(0.57-0.81)$ & 89.41 & $0.78(0.67-0.88)$ \\
\hline & IL2 & & & & 106.53 & $0.62(0.44-0.81)$ & 103.28 & $0.69(0.55-0.82)$ \\
\hline IL-6 & & & & & 107.26 & $0.56(0.37-0.76)$ & 91.01 & $0.76(0.65-0.87)$ \\
\hline $2 \mathrm{Bion}$ & & & & & & & & \\
\hline & & & SERPINA3 & AZGP1 & 100.68 & $0.73(0.61-0.84)$ & 104.08 & $0.69(0.57-0.81)$ \\
\hline & & CRP & & AZGP1 & 93.82 & $0.77(0.67-0.88)$ & 92.81 & $0.78(0.68-0.89)$ \\
\hline & IL2 & & & AZGP1 & 101.90 & $0.72(0.61-0.84)$ & 99.83 & $0.74(0.63-0.86)$ \\
\hline IL-6 & & & & AZGP1 & 102.97 & $0.71(0.59-0.83)$ & 91.80 & $0.79(0.69-0.89)$ \\
\hline & & CRP & SERPINA3 & & 101.49 & $0.71(0.60-0.83)$ & 93.44 & $0.78(0.67-0.89)$ \\
\hline & IL2 & & SERPINA3 & & 109.35 & $0.65(0.50-0.79)$ & 103.06 & $0.72(0.60-0.85)$ \\
\hline IL-6 & & & SERPINA3 & & 110.15 & $0.60(0.47-0.72)$ & 93.48 & $0.77(0.67-0.88)$ \\
\hline & IL2 & CRP & & & 100.97 & $0.73(0.61-0.85)$ & 87.33 & $0.82(0.72-0.92)$ \\
\hline IL-6 & & CRP & & & 101.43 & $0.70(0.58-0.82)$ & 87.16 & $0.82(0.72-0.92)$ \\
\hline IL-6 & IL2 & & & & 109.82 & $0.63(0.45-0.80)$ & 92.50 & $0.77(0.67-0.88)$ \\
\hline 3 Bion & & & & & & & & \\
\hline & & CRP & SERPINA3 & AZGP1 & 97.90 & $0.78(0.68-0.88)$ & 96.83 & $0.79(0.69-0.89)$ \\
\hline & IL2 & & SERPINA3 & AZGP1 & 103.30 & $0.72(0.64-0.86)$ & 101.78 & $0.76(0.64-0.87)$ \\
\hline & IL2 & CRP & & AZGP1 & 95.84 & $0.79(0.69-0.89)$ & 89.95 & $0.82(0.73-0.92)$ \\
\hline & IL2 & CRP & SERPINA3 & & 103.43 & $0.74(0.63-0.86)$ & 91.11 & $0.82(0.73-0.92)$ \\
\hline IL-6 & & & SERPINA3 & AZGP1 & 104.55 & $0.73(0.62-0.85)$ & 95.07 & $0.80(0.70-0.90)$ \\
\hline IL-6 & & CRP & & AZGP1 & 97.27 & $0.78(0.68-0.88)$ & 90.46 & $0.83(0.73-0.92)$ \\
\hline IL-6 & & CRP & SERPINA3 & & 104.19 & $0.73(0.61-0.84)$ & 91.34 & $0.82(0.72-0.92)$ \\
\hline IL-6 & IL2 & & & AZGP1 & 105.27 & $0.73(0.62-0.85)$ & 91.42 & $0.81(0.72-0.91)$ \\
\hline IL-6 & IL2 & & SERPINA3 & & 112.82 & $0.66(0.51-0.80)$ & 94.58 & $0.80(0.69-0.90)$ \\
\hline IL-6 & IL2 & CRP & & & 103.60 & $0.74(0.61-0.86)$ & 87.11 & $0.84(0.75-0.93)$ \\
\hline 4 Bion & & & & & & & & \\
\hline & IL2 & CRP & SERPINA3 & AZGP1 & 99.67 & $0.80(0.70-0.90)$ & 93.74 & $0.83(0.74-0.93)$ \\
\hline IL-6 & & CRP & SERPINA3 & AZGP1 & 101.20 & $0.79(0.69-0.89)$ & 94.68 & $0.83(0.74-0.93)$ \\
\hline IL-6 & IL2 & & SERPINA3 & AZGP1 & 107.17 & $0.75(0.64-0.86)$ & 94.60 & $0.82(0.73-0.92)$ \\
\hline IL-6 & IL2 & CRP & & AZGP1 & 99.01 & $0.80(0.70-0.90)$ & 89.47 & $0.85(0.76-0.94)$ \\
\hline IL-6 & IL2 & CRP & SERPINA3 & & 105.65 & $0.76(0.65-0.88)$ & 91.03 & $0.84(0.75-0.94)$ \\
\hline 5 Bion & & & & & & & & \\
\hline IL-6 & IL2 & CRP & SERPINA3 & AZGP1 & 102.59 & $0.81(0.71-0.91)$ & 93.48 & $0.86(0.77-0.94)$ \\
\hline
\end{tabular}

Note SERPINA3 = alpha-1 antichymotrypsin; CRP = C-reactive protein; IL = interleukin; POD2 = postoperative day 2; PREOP = preoperative; AZGP1 = zincalpha2-glycoprotein. The two bolded rows indicate the "best" PREOP and "best" POD2 models as determined from the lowest Bayesian information criteria (BIC) value and highest c-statistic. When these two criteria did not agree with each other, we prioritized the BIC. BIC and c-statistic presented for average of 1,000 bootstrapped samples based on the 75 matched pairs.

SERPINA3 is a serine protease inhibitor and inflammatory protein that has been associated with delirium and Alzheimer's Disease (24,25). Although SERPINA3 was identified but not included in either our PREOP or POD2 models, additional work in a larger sample may underscore its importance in delirium. Taken together, our findings provide strong support for the role of inflammation in delirium pathophysiology.

The current study includes several notable strengths. First, the availability of multiple time points of blood draw allowed for identification (and validation) of proteins that follow two distinct, previously described biomarker patterns-risk markers and disease markers, for postoperative delirium (13). Second, we used a rigorous multi-stage process that employed a proteomics approach followed by ELISA confirmation and independent validation to identify proteins consistently associated with postoperative delirium. This multi-stage approach provides protection for false discovery, which is equivalent to adjusting the $p$-value in a single stage approach. Third, multivariable modeling enabled us to identify the best, most parsimonious multi-protein model of delirium at PREOP and POD2representing a novel contribution to the literature on delirium pathophysiology.

Some limitations of the current study warrant mention. First, we recognize the limitation of using plasma measures and unavailability of CSF for identifying proteins associated with postoperative delirium. Second, we acknowledge that our examination of a single postoperative time point does not enable consideration of temporal changes post-surgery. Third, variations in time between the PREOP blood draw time point and surgery date could have introduced variability in this measure. Fourth, our inclusion of different surgery types (orthopedic, vascular, and gastrointestinal) may influence the observed associations between our identified proteins and delirium; however, examining the associations in a subset of orthopedic patients (66 matched pairs) yielded similar findings. Fifth, we considered how our use of combined CAM and chart-defined delirium 
A)

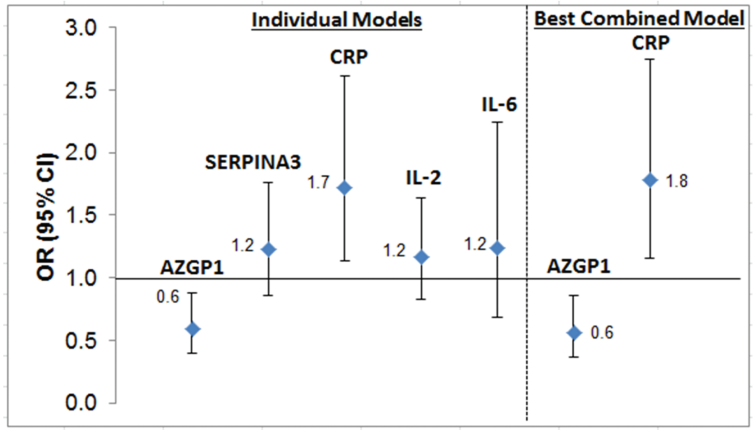

B)

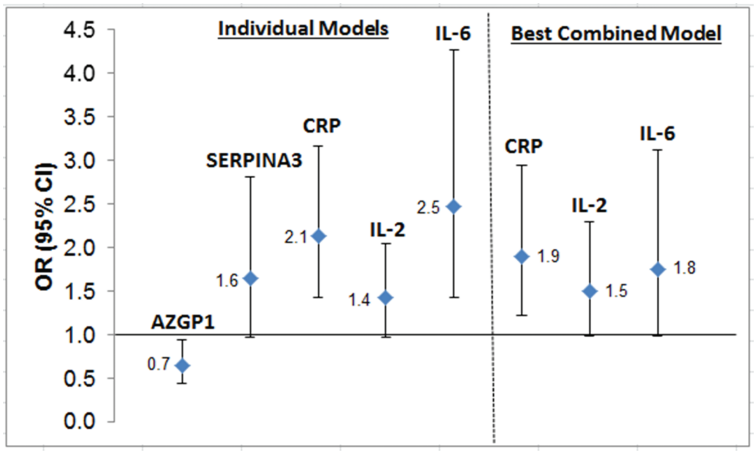

Figure 2. Association between biomarkers and delirium from models of individual biomarkers and of the "best combined model" for PREOP (A) and POD2 (B). SERPINA3 = alpha-1 antichymotrypsin; $\mathrm{Cl}=$ confidence interval; $\mathrm{CRP}=\mathrm{C}$-reactive protein; $\mathrm{IL}=$ interleukin; $\mathrm{OR}=$ odds ratio; POD2 = postoperative day; $\mathrm{PREOP}=$ preoperative; $\mathrm{AZGP1}=$ zinc-alpha2 glycoprotein. For the best combined model for PREOP and POD2, each protein was included as a separate variable into the analytic model.

may influence our observed associations. Our sensitivity analyses confirmed that our reported findings hold when examining a subset of only CAM-defined delirium cases (and their matched no-delirium controls). Finally, our exclusion of older adults with dementia limits the generalizability of our findings, but also eliminates a potential confounder. Future work in populations with different clinical populations (eg, older patients not undergoing surgery and those with dementia) will expand our knowledge of delirium pathophysiology.

Our use of matched case-control samples did not fully allow for advanced statistical analyses such as the use of structural equation modeling (SEM) to determine the direction of protein associations with delirium and with one another. We are currently measuring the proteins identified in this study across the entire SAGES cohort, which will provide sufficient information to further explore these relationships using pathway analyses and will be the next step in replicating our findings. This future work will also include examination of protein-protein interactions, which is required to further understand how the protein milieu influences development of delirium. In the future, we anticipate validating our findings in an entirely independent new cohort. This future planned work will, in part, address the potential levels of uncertainty surrounding our multistage process of identifying, confirming, and validating our top set of delirium-associated proteins.

In summary, we found that CRP, AZGP1, and SERPINA3 were associated with postoperative delirium, and the "best" multi-protein models of delirium included CRP and AZGP1 at PREOP and IL-6, IL-2, and CRP on POD2. Our findings provide the foundation for the eventual clinical use of these multi-protein signatures in identifying patients at-risk for delirium, for monitoring its course, and for informing design of pathophysiologically based interventions. Future work using alternative proteomics platforms, plus lipidomic and metabolomic methodologies will enhance our understanding of the complex and dynamic pathophysiology of delirium.

\section{Funding}

This research was supported by National Institute on Aging grants (R01AG051658， P01AG031720， K07AG041835， R01AG044518, R01AG030618, K24AG035075, R21AG048600, R01AG041274, and R24AG054259), National Center for Complementary and Integrative Health (T32AT000051), and the Charles A. King Trust Postdoctoral Research Fellowship Program, Bank of America, N.A., Co-Trustee. Dr Inouye holds the Milton and Shirley F. Levy Family Chair. Dr Kuchel holds the Travelers Chair in Geriatrics and Gerontology. The authors gratefully acknowledge the contributions of the patients, family members, nurses, physicians, staff members, and members of the Executive Committee who participated in the Successful Aging after Elective Surgery (SAGES) Study.

\section{Conflict of Interest}

The authors report no conflicts of interest. Dr Edward Marcantonio serves on the Editorial Board of the Journal of Gerontology: Medical Sciences.

\section{References}

1. Inouye SK, Westendorp RG, Saczynski JS. Delirium in elderly people. Lancet. 2014;383:911-922. doi:10.1016/S0140-6736(13)60688-1

2. Marcantonio ER. Postoperative delirium: a 76-year-old woman with delirium following surgery. JAMA. 2012;308:73-81. doi:10.1001/jama.2012.6857

3. Davis DH, Muniz Terrera G, Keage H, et al. Delirium is a strong risk factor for dementia in the oldest-old: a population-based cohort study. Brain. 2012;135:2809-2816. doi:10.1093/brain/aws190

4. Witlox J, Eurelings LS, de Jonghe JF, Kalisvaart KJ, Eikelenboom P, van Gool WA. Delirium in elderly patients and the risk of postdischarge mortality, institutionalization, and dementia: a meta-analysis. JAMA. 2010;304:443-451. doi:10.1001/jama.2010.1013

5. Maldonado JR. Neuropathogenesis of delirium: review of current etiologic theories and common pathways. Am J Geriatr Psychiatry. 2013;21:11901222. doi:10.1016/j.jagp.2013.09.005

6. Vasunilashorn SM, Ngo L, Inouye SK, et al. Cytokines and postoperative delirium in older patients undergoing major elective surgery. J Gerontol A Biol Sci Med Sci. 2015;70:1289-1295. doi:10.1093/gerona/glv083

7. Dillon ST, Vasunilashorn SM, Ngo L, et al. Higher C-reactive protein levels predict postoperative delirium in older patients undergoing major elective surgery: a longitudinal nested case-control study. Biol Psychiatry. 2017;81:145-153. doi:10.1016/j.biopsych.2016.03.2098

8. van Munster BC, Aronica E, Zwinderman AH, Eikelenboom P, Cunningham C, de Rooij SE. Neuroinflammation in delirium: a postmortem case-control study. Rejuvenation Res. 2011;14:615-622. doi:10.1089/ rej.2011.1185

9. Cerejeira J, Firmino H, Vaz-Serra A, Mukaetova-Ladinska EB. The neuroinflammatory hypothesis of delirium. Acta Neuropathol. 2010;119:737754. doi:10.1007/s00401-010-0674-1

10. Schmitt EM, Marcantonio ER, Alsop DC, et al.; SAGES Study Group. Novel risk markers and long-term outcomes of delirium: the successful aging after elective surgery (SAGES) study design and methods. J Am Med Dir Assoc. 2012;13:818.e1-818.e10. doi:10.1016/j.jamda.2012.08.004

11. Schmitt EM, Saczynski JS, Kosar CM, et al.; SAGES Study Group. The Successful Aging after Elective Surgery (SAGES) Study: cohort description and data quality procedures. J Am Geriatr Soc. 2015;63:2463-2471. doi:10.1111/jgs.13793

12. Jones RN, Rudolph JL, Inouye SK, et al. Development of a unidimensional composite measure of neuropsychological functioning in older cardiac surgery patients with good measurement precision. J Clin Exp Neuropsychol. 2010;32:1041-1049. doi:10.1080/13803391003662728 
13. Marcantonio ER, Rudolph JL, Culley D, Crosby G, Alsop D, Inouye SK. Serum biomarkers for delirium. J Gerontol A Biol Sci Med Sci. 2006;61:1281-1286. doi:10.1093/gerona/61.12.1281

14. Saczynski JS, Kosar CM, Xu G, et al. A tale of two methods: chart and interview methods for identifying delirium. I Am Geriatr Soc. 2014;62:518-524. doi:10.1111/jgs.12684

15. Inouye SK, van Dyck CH, Alessi CA, Balkin S, Siegal AP, Horwitz RI Clarifying confusion: the confusion assessment method. A new method for detection of delirium. Ann Intern Med. 1990;113:941-948.

16. Ngo LH, Inouye SK, Jones RN, et al. Methodologic considerations in the design and analysis of nested case-control studies: association between cytokines and postoperative delirium. BMC Med Res Methodol. 2017;17:88. doi:10.1186/s12874-017-0359-8

17. Ross PL, Huang YN, Marchese JN, Williamson B, Parker K, Hattan S, et al. Multiplexed protein quantitation in Saccharomyces cerevisiae using amine-reactive isobaric tagging reagents. Mol Cell Proteomics. 2004;3:1154-1169. doi:10.1074/mcp.M400129-MCP200

18. Burnham KP, Anderson DR. Model Selection and Multimodel Inference: A Practical Information-Theoretic Approach, 2nd ed. New York: Springer; 2012.

19. Lee HJ, Hwang DS, Wang SK, Chee IS, Baeg S, Kim JL. Early assessment of delirium in elderly patients after hip surgery. Psychiatry Investig. 2011;8:340-347. doi:10.4306/pi.2011.8.4.340
20. Hassan MI, Waheed A, Yadav S, Ingh TP, Ahmad F. Zinc $\alpha 2$-glycoprotein: a multidisciplinary protein. Mol Cancer Res. 2008;6:892-906. doi:10.1158/1541-7786.MCR-07-2195

21. Westhoff D, Witlox J, van Aalst C, et al. Preoperative protein profiles in cerebrospinal fluid in elderly hip fracture patients at risk for delirium: a proteomics and validation study. BBA Clin. 2015;4:115-122. doi:10.1016/j.bbacli.2015.10.002

22. Mracek T, Gao D, Tzanzvari T, et al. Downregulation of zinc-alpha2-glycoprotein in adipose tissue and liver of obese ob/ob mice and by tumour necrosis factor-alpha in adipocytes. J Endocrinol. 2010;204:165-72. doi:10.1677/JOE-09-0299

23. Welters ID, Binc C, Ding C, Leuwer M, Hall AM. Circulating anti-inflammatory adipokines high molecular weight adiponectin and Zinc$\alpha 2$-glycoprotein (ZAG) are inhibited in early sepsis, but increase with clinical recovery: a pilot study. BMC Anesthesiol. 2014;14:124 doi:10.1186/1471-2253-14-124

24. Poljak A, Hill M, Hall RJ, et al. Quantitative proteomics of delirium cerebrospinal fluid. Transl Psychiatry. 2014;4:e477. doi:10.1038/ tp.2014.114

25. Padmanabhan J, Levy M, Dickson DW, Potter H. Alpha 1-antichymotrypsin, an inflammatory protein overexpressed in Alzheimer's disease brain, induces tau phosphorylation in neurons. Brain 2006;129:3020 3034. doi:10.1093/brain/awl255 